\title{
INTRINSIC FORM OF THE CHARACTERISTIC RELATIONS IN THE STEADY SUPERSONIC FLOW OF A COMPRESSIBLE FLUID*
}

\author{
BY \\ N. COBURN \\ University of Michigan
}

1. Introduction. The purpose of this paper is to express the characteristic relations for the steady, supersonic, three-dimensional, rotational and irrotational motion of a polytropic gas in intrinsic form and to apply these relations to the study of a class of space Beltrami motions. This class of motions is characterized by the fact that one family of the characteristic surfaces consists of $\infty^{1}$ parallel planes.

To obtain intrinsic forms of the characteristic relations, two techniques must be used. First, the equations of motion, continuity, and energy must be expressed in terms of a set of characteristic variables. Secondly, these must be reduced to intrinsic form by expressing the partial derivatives of the components of the velocity vector in terms of the directional derivatives of the magnitude of the velocity vector, $q$, and the sound speed, $c$, and the curvatures associated with the characteristic manifolds.

The class of fluid motions to be studied is characterized by the fact that one family of $\infty^{1}$ characteristic surfaces consists of planes, $z=$ constant. If the additional assumption is made that the bicharacteristics and their orthogonal trajectories determine two families of cylindrical surfaces with generators parallel to the $z$-axis, then it is shown that these surfaces are right circular cylinders with $o z$ as axis; the bicharacteristics are concentric circles; the stream lines are helices along which $q$ (or $M$, the Mach number) is constant.

2. The basic relation in terms of characteristic variables. Let $x^{i}, j=1,2,3$, denote a Cartesian orthogonal coordinate system in Euclidean three-space and let us denote partial derivatives by the symbolism

$$
\partial_{i} \equiv \frac{\partial}{\partial x^{i}} .
$$

In a Cartesian orthogonal coordinate system, covariant and contravariant quantities are equivalent. However, in order to use the Einstein summation convention of summing on repeated lower and upper indices, we shall use these two equivalent quantities.

First, we consider the equations of continuity, motion, and energy in a Cartesian orthogonal coordinate system. Let $\rho$ denote the specific density and $v^{i}$ denote the velocity vector, then the equation of continuity is

$$
v^{i} \partial_{i} \rho+\rho \partial_{i} v^{i}=0 .
$$

For non-isentropic flows of a polytropic gas, the equations of motion may be written in the form [1]

$$
\gamma \rho v^{i} \partial_{j} v_{k}+\partial_{k}\left(\rho c^{2}\right)=0,
$$

${ }^{*}$ Received April 17, 1956; revised manuscript received July 5, 1956. The author is indebted to the Office of Ordnance Research, U. S. Army, for financial aid during this research, which was administered by the Engineering Research Institute of the University of Michigan. 
where $c$ is the local sound speed which may be defined in terms of the pressure, $p$, and the specific entropy $S$ by

$$
c^{2}=\left(\frac{\partial p}{\partial \rho}\right)_{s}
$$

and $\gamma$ is the constant of the gas law

$$
p=\rho^{\gamma} f(S) .
$$

In addition, we shall use as energy equation the Bernoulli relation

$$
v^{i} \partial_{i}\left(\frac{c^{2}}{\gamma-1}+\frac{q^{2}}{2}\right)=0,
$$

where $q^{2}=v^{i} v_{i}$ is the magnitude squared of the velocity vector.

By definition, the characteristic manifolds of the system (2.1), (2.2), and (2.3) are those surfaces along which discontinuities in the derivatives, $\partial_{i} \rho, \partial_{i} c, \partial_{j} v_{k}$, can occur. Using the methods of a previous paper [2], these surfaces can be easily determined. However, these methods are not pertinent to the present problem; hence, the procedure which follows will be by analogy. By eliminating $\rho$ between (2.1) and (2.2) and then by eliminating $\partial_{i} c^{2}$ in the resulting equation through use of (2.3), we obtain the following relation

$$
\left(v^{i} v^{k}-c^{2} g^{i k}\right) \partial_{j} v_{k}=0,
$$

where $g^{i k}$ represents the metric tensor. Thus, the same basic equation (2.4) is valid in the non-isentropic as well as in the isentropic case [3].

If the symmetric tensor $a^{i k}$ is defined by

$$
a^{i k}=v^{i} v^{k}-c^{2} g^{i k}
$$

then for isentropic flows the characteristic manifolds of (2.4) are determined by

$$
a^{i k} n_{j} n_{k}=0,
$$

where $n_{i}$ is a unit vector orthogonal to one family of characteristic surfaces. It can be shown that (2.6) determines the characteristic manifolds in the non-isentropic case [2]. From the known theory of characteristic manifolds [4], it follows that

$$
v^{i}=c n^{i}+b t^{i}
$$

where $t^{i}$ is the unit tangent vector to the bicharacteristic curves and

$$
b=\left(q^{2}-c^{2}\right)^{1 / 2} \text {. }
$$

We shall require that both $b$ and $c$ be positive. However, we shall not fix the sense of $t^{i}, n^{i}$. This means that we shall be able to use either nappe of the normal or bicharacteristic cones.

By use of (2.5), (2.7), we find that

$$
\begin{aligned}
& a^{i k} n_{k}=b c t^{i}, \\
& a^{i k} t_{k}=b c n^{i}+\left(q^{2}-2 c^{2}\right) t^{i} .
\end{aligned}
$$

Secondly, we shall introduce a few basic relations from differential geometry [5]. 
Since $n^{i}$ is a unit vector orthogonal to $\infty^{1}$ surfaces, we may write

$$
\partial_{i} n_{k}=s_{i k}+n_{i} u_{k}, \quad s_{i k} n^{k}=u_{k} n^{k}=0,
$$

where $s_{i k}$ is the symmetric second fundamental tensor of the $\infty^{1}$ surfaces orthogonal to $n_{j}$ and $u_{k}$ is the curvature vector of the $n_{i}$ congruence of curves. Further, let $p_{i}$ denote a unit vector field which is orthogonal to both $t_{i}$ and $n_{i}$ so that $p_{i}, t_{i}$, and $n_{i}$ form an ordered orthogonal right hand triple at each point. The curvature vector of the $p_{i}$ congruence of curves will be denoted by $w_{i}$, and the curvature vector of the $t_{i}$ congruence by $m_{i}$ so that

$$
\begin{aligned}
w_{i} & =p^{k} \partial_{k} p_{i} . \\
m_{i} & =t^{k} \partial_{k} t_{j} .
\end{aligned}
$$

From the decomposition of the metric tensor

$$
g^{i k}=t^{i} t^{k}+n^{i} n^{k}+p^{i} p^{k}
$$

we obtain the relation

$$
g^{i k} \partial_{j} t_{k}=t^{i} t^{k} \partial_{j} t_{k}+n^{i} n^{k} \partial_{i} t_{k}+p^{i} p^{k} \partial_{j} t_{k} .
$$

It is easily seen that

$$
t^{i} t^{k} \partial_{i} t_{k}=0, \quad n^{i} n^{k} \partial_{j} t_{k}=-t_{k} u^{k}, \quad p^{j} p^{k} \partial_{j} t_{k}=-t_{k} w^{k} .
$$

Thus, we may express (2.13) in the form

$$
g^{i k} \partial_{i} t_{k}=-t_{k}\left(u^{k}+w^{k}\right) \text {. }
$$

In this paragraph, the relations (2.7), (2.11), (2.15) will be used to express the basic relation (2.4) in terms of the rates of change of $q$ and $c$ with respect to displacements along $t^{i}$ and $n^{i}$, and in terms of the curvatures $s_{i k}, u_{i}, w_{i}$. The following notation for directional derivatives will be used

$$
\frac{\partial}{\partial t} \equiv t^{i} \partial_{i}, \quad \frac{\partial}{\partial n} \equiv n^{i} \partial_{i}, \quad \frac{\partial}{\partial p} \equiv p^{i} \partial_{i} .
$$

Thus, $\partial / \partial t$ represents rate of change with respect to displacement along $t^{i}$, and similar interpretations are valid for $\partial / \partial n, \partial / \partial p$. By differentiation of (2.7), we find

$$
\partial_{j} v_{k}=c \partial_{i} n_{k}+b \partial_{i} t_{k}+n_{k} \partial_{j} c+t_{k} \partial_{i} b .
$$

Multiplying (2.16) by $a^{i k}$ and using (2.9), (2.10), we obtain the relation

$$
a^{i k} \partial_{j} v_{k}=c a^{i k} \partial_{j} n_{k}+b a^{i k} \partial_{j} t_{k}+c b\left(\frac{\partial c}{\partial t}+\frac{\partial b}{\partial n}\right)+\left(q^{2}-2 c^{2}\right) \frac{\partial b}{\partial t} .
$$

To evaluate the term $a^{i k} \partial_{i} n_{k}$ in equation (2.17), we use (2.5), (2.7) and find

$$
a^{i k}=c^{2}\left(n^{i} n^{k}-g^{i k}-t^{i} t^{k}\right)+q^{2} t^{i} t^{k}+c b\left(t^{i} n^{k}+n^{i} t^{k}\right) .
$$

Forming the scalar product of (2.11), (2.18), we obtain

$$
a^{i k} \partial_{i} n_{k}=c b t^{k} u_{k}+b^{2} s_{j k} t^{i} t^{k}-c^{2} M^{*}
$$

where $M^{*}$ is the mean curvature of the characteristic surfaces

$$
M^{*}=g^{i j} s_{i k} .
$$


To determine the term $a^{i k} \partial_{i} t_{k}$ of equation (2.17), we use (2.18), (2.14), (2.15) and find

$$
a^{i k} \partial_{i} t_{k}=-c b s_{i k} t^{i} t^{k}+c^{2} t_{k} w^{k} \text {. }
$$

If (2.19), (2.20) are substituted into (2.17) and the left hand side of the latter equation is equated to zero, we obtain the desired intrinsic form of the basic relation (2.4)

$$
c b\left(\frac{\partial c}{\partial t}+\frac{\partial b}{\partial n}\right)+\left(q^{2}-2 c^{2}\right) \frac{\partial b}{\partial t}+c^{2} b t_{k}\left(u^{k}+w^{k}\right)-c^{8} M^{*}=0 .
$$

3. The intrinsic conditions for rotational (or irrotational) motion in terms of characteristic variables. If $e^{i j k}$ denotes the permutation tensor, then the vorticity vector $\omega^{i}$ is defined by

$$
\omega^{i}=e^{i j k} \partial_{i} v_{k} .
$$

Since the ordered triad $p_{i}, t_{i}, n_{i}$ forms a right hand system, the following formulae are obtained by forming the scalar products of (3.1) with the vectors $t_{i}, n_{i}, p_{i}$, respectively

$$
\begin{aligned}
\omega^{i} t_{i} & =\left(n^{i} p^{k}-n^{k} p^{i}\right) \partial_{i} v_{k}, \\
\omega^{i} n_{i} & =\left(p^{i} t^{k}-p^{k} t^{i}\right) \partial_{i} v_{k}, \\
\omega^{i} p_{i} & =\left(t^{i} n^{k}-t^{k} n^{i}\right) \partial_{i} v_{k} .
\end{aligned}
$$

A lengthy but direct computation by use of (2.11), (2.12), (2.16) shows that (3.2) may be written as

$$
\begin{aligned}
& \omega^{i} t_{i}=c p^{k} u_{k}-\frac{\partial c}{\partial p}+b\left(n^{i} p^{k}-n^{k} p^{i}\right) \partial_{i} t_{k}, \\
& \omega^{i} n_{i}=\frac{\partial b}{\partial p}-b p^{k} m_{k} \\
& \omega^{i} p_{i}=\frac{\partial c}{\partial t}-\frac{\partial b}{\partial n}-b s_{i k} t^{i} t^{k}-c t_{k} u^{k} .
\end{aligned}
$$

The Eqs. (3.3) determine the intrinsic form of the conditions for irrotational motion.

Now, we consider a form of the equations of motion which contain the vorticity [6]

$$
\partial_{i} h_{0}-T \partial_{i} S=e_{i i k} v^{i} \omega^{k} \text {. }
$$

Here, $T$ is the absolute temperature, $S$ is the specific entropy, and $h_{0}$ is the stagnation enthalpy

$$
h_{0}=\frac{c^{2}}{\gamma-1}+\frac{q^{2}}{2},
$$

where $\gamma$ is the ratio of the specific heats of the polytropic gas. Since the stagnation enthalpy is constant along a stream line (see 2.3), with the aid of (2.7) we find that

$$
c \frac{\partial h_{0}}{\partial n}+b \frac{\partial h_{0}}{\partial t}=0
$$

and $S$ satisfies a similar relation.

To express (3.4) in intrinsic form, the scalar product of this relation with the vectors $t_{i}, n_{i}$, and $p_{i}$ will be found. Using (2.7), (3.1), and (3.3), the following formulae are 
obtained

$$
\begin{gathered}
\frac{\partial h_{0}}{\partial t}-T \frac{\partial S}{\partial t}=c\left(\frac{\partial c}{\partial t}-\frac{\partial b}{\partial n}-b s_{j k} t^{i} t^{k}-c t^{k} u_{k}\right) \\
\frac{\partial h_{0}}{\partial n}-T \frac{\partial S}{\partial n}=-b\left(\frac{\partial c}{\partial t}-\frac{\partial b}{\partial n}-b s_{j k} t^{i} t^{k}-c t^{k} u_{k}\right) \\
\frac{\partial h_{\mathrm{n}}}{\partial p}-T \frac{\partial S}{\partial p}=q \frac{\partial q}{\partial p}-\left(b^{2} m_{k}+c^{2} u_{k}\right) p^{k}-c b n^{j} p^{k}\left(\partial_{j} t_{k}-\partial_{k} t_{j}\right)
\end{gathered}
$$

Evidently, (3.8) is a consequence of (3.6), (3.7).

The above equations can be described by saying that they form a system consisting of three equations (3.7), (3.9), and (2.21) in the unknowns

$$
\frac{\partial q}{\partial t}, \quad \frac{\partial q}{\partial n}, \quad \frac{\partial q}{\partial p},
$$

where $h_{0}$ and $S$ are prescribed functions which are constant along a stream line. 'The sound speed, $c$, is determined as a function of the magnitude of the velocity, $q$, by the Bernoulli relation (3.5). Thus, the roles of $c$ and $q$ may be interchanged. In the applications, the stream lines are unknown. Hence, the problem is to determine the functions $h_{0}, S$, and $q$ so that two relations of the type (3.6) are satisfied (one equation in the derivatives of $h_{0}$ and the other in the derivatives of $S$ ) and also Eqs. (3.7), (3.9) and (2.21) are valid.

The curvature term,

$$
K=n^{i} p^{k}\left(\partial_{i} t_{k}-\partial_{k} t_{i}\right),
$$

in the right hand side of (3.9) will be briefly considered. Through use of (2.11), this term may be written as

$$
K=-\left(n^{i} t^{k} \partial_{i} p_{k}-t^{i} p^{k} \partial_{k} n_{i}\right)=-n^{i} t^{k} \partial_{i} p_{k}+s_{k i} t^{i} p^{k}
$$

If the unit vector field $p_{i}$ is orthogonal to $\infty^{1}$ surfaces, as is the case in plane and axialsymmetric flow, then

$$
\partial_{i} p_{k}=r_{i k}+p_{i} w_{k}, \quad p^{k} r_{i k}=p^{k} w_{k}=0,
$$

where $r_{j k}$ is the symmetric second fundamental tensor of these surfaces and $w_{k}$ is the curvature vector of the $p_{i}$ congruence (see 2.12). In this case, (3.11) reduces to

$$
K=-r_{i k} n^{i} t^{k}+s_{i k} t^{i} p^{k} \text {. }
$$

For the special cases of plane and axial-symmetric flow, $K$ vanishes. In both cases, $p_{i}$ is orthogonal to $\infty^{1}$ planes and hence $r_{j k}=0$ and $p_{i}, t_{i}$ are in the principal directions of the surfaces orthogonal to $n_{i}$ [7]. The $\infty^{2}$ planes are parallel in plane flow; they intersect in a common line in axially symmetric flow.

4. Canonical form of the system (2.21), (3.7), and (3.9). In this section the equations (2.21), (3.7), and (3.9) will be written so that one equation contains only the directional derivative, $\partial / \partial t$, a second equation contains only the directional derivative $\partial / \partial n$, and the final equation contains only the directional derivative, $\partial / \partial p$.

If (3.7) is multiplied by $b$ and the resulting equation is added to (2.21) the following relation is obtained. 


$$
b^{2} \frac{\partial}{\partial t} \frac{q^{2}}{b}=-c^{2} b t_{k} w^{k}+b^{2} c s_{i k} t^{i} t^{k}+c^{3} M^{*}+b\left(\frac{\partial h_{0}}{\partial t}-T \frac{\partial S}{\partial t}\right)
$$

For $q>c$, the equation (4.1) is the first of the desired canonical relations.

To determine the second relation of desired canonical system, we multiply (3.7) by $b$ and subtract from (2.21). We find that

$$
\begin{aligned}
2 c b \frac{\partial b}{\partial n}+\left(q^{2}-2 c^{2}\right) \frac{\partial b}{\partial t}+c^{2} b t_{k}\left(2 u^{k}+w^{k}\right)-c^{3} M^{*}+c b^{2} s_{i k} t^{i} t^{k} & \\
= & =-b\left(\frac{\partial h_{0}}{\partial t}-T \frac{\partial S}{\partial t}\right)
\end{aligned}
$$

To evaluate the second term of the left hand side of (4.2), we use (3.5), (3.7) and obtain

$$
(\gamma-1) b \frac{\partial b}{\partial t}=-2 \frac{\partial h_{0}}{\partial t}+(\gamma+1)\left[T \frac{\partial S}{\partial t}-c \frac{\partial b}{\partial n}-c b s_{j k} t^{i} t^{k}-c^{2} t_{k} u^{k}\right]
$$

Converting $\partial h_{0} / \partial t, \partial S / \partial t$ into $\partial h_{0} / \partial n, \partial S / \partial n$ by use of (3.6) we find that (4.3) reduces to

$$
(\gamma-1) b^{2} \frac{\partial b}{\partial t}=2 c \frac{\partial h_{0}}{\partial n}-(\gamma+1)\left[c T \frac{\partial S}{\partial n}+b c \frac{\partial b}{\partial n}+c b^{2} s_{i k} t^{i} t^{k}+c^{2} b t_{k} u^{k}\right]
$$

Substituting (4.4) into (4.2) and converting $\partial h_{0} / \partial t, \partial S / \partial t$ of (4.2) into $\partial h_{0} / \partial n, \partial S / \partial n$ by use of (3.6), we obtain the second canonical relation for $q>c$.

$$
\begin{aligned}
b\left[(\gamma-3) q^{2}+4 c^{2}\right] \frac{\partial b}{\partial n} & =\left[2 q^{2}-(\gamma+3) c^{2}\right] b^{2} s_{j k} t^{i} t^{k}+(\gamma-1) b^{2} c^{2} M^{*} \\
& -c b\left[(\gamma-3) q^{2}+4 c^{2}\right] t_{k} u^{k}-(\gamma-1) b^{3} c t_{k} w^{k} \\
+ & {\left[(\gamma-3) q^{2}-(\gamma-5) c^{2}\right] \frac{\partial h_{0}}{\partial n}+\left[2 q^{2}-(\gamma+3) c^{2}\right] T \frac{\partial S}{\partial n} }
\end{aligned}
$$

Evidently, (3.9) is the third equation of the desired canonical system. This equation can be written in the form

$$
q \frac{\partial q}{\partial p}=\frac{\partial h_{0}}{\partial p}-T \frac{\partial S}{\partial p}+\left(b^{2} m_{k}+c^{2} u_{k}\right) p^{k}+c b K
$$

where $K$ is the curvature defined in (3.10).

5. A class of space flows. For plane flows, the two families of characteristic surfaces can be chosen to be right cylinders with parallel generators which are perpendicular to the plane of the flow. Here, we consider the case where one family of characteristic surfaces are parallel planes. We shall show that this condition defines a family of space flows.

We recall that in Sec. 3, we assumed that the ordered triad of unit vectors $p_{i}, t_{i}, n_{i}$ forms a right hand system. If the characteristic planes are assumed to be perpendicular to the $z$-axis of an $x, y, z$ Cartesian orthogonal coordinate system, then $n_{i}$ can be chosen to be sensed either in the positive $z$-direction or in the negative $z$-direction. In the first case, the ordered pair, $p_{i}, t_{j}$ forms a right hand system when considered as part of the ordered triple $p_{i}, t_{i}$, and the positive $z$-direction. The second case leads to nothing new. Hence only the first case will be considered.

In any plane orthogonal to the $z$-axis, we introduce two families of parameter curves: $\alpha=$ variable along $t_{j}, \beta=$ variable along $p_{i}$. Arc length will be positive when measured 
in the directions of $t_{i}, p_{i}$, respectively. Every family of $\infty^{1}$ curves, $\alpha=$ variable $(z=$ constant, $\beta=$ constant), generates a surface. Any surface $\beta=$ constant will be chosen so that it consists of $\infty^{1}$ curves, $\alpha=$ variable, lying in $\infty^{1}$ planes, $z=$ constant. We choose the surfaces, $\alpha=$ constant, in a similar manner. The third family of surfaces in our new coordinate system will be the planes, $z=\delta=$ constant. The transformation equations relating $\alpha, \beta, \delta$ to $x, y, z$ are of the form

$$
x=x(\alpha, \beta, \delta), \quad y=y(\alpha, \beta, \delta), \quad z=\delta .
$$

In any plane, $\delta=$ constant, the arc length element is

$$
d s^{2}=(A d \alpha)^{2}+(B d \beta)^{2},
$$

where $A, B$ are functions of $\alpha, \beta, \delta$. If $\theta(\alpha, \beta, \delta)$ denotes the angle between the $x$-axis and $t_{i}$ then the unit vectors $t_{i}, p_{i}$ have Cartesian orthogonal components

$$
\begin{gathered}
t_{i}:(\cos \theta, \sin \theta, 0), \\
p_{i}:(\sin \theta,-\cos \theta, 0) .
\end{gathered}
$$

Thus, we find that

$$
\begin{array}{ll}
\frac{\partial x}{\partial \alpha}=A \cos \theta, & \frac{\partial y}{\partial \alpha}=A \sin \theta \\
\frac{\partial x}{\partial \beta}=B \sin \theta, & \frac{\partial y}{\partial \beta}=-B \cos \theta .
\end{array}
$$

In the case of curves lying in the planes, $z=$ constant, the sense of the principal normal vector is chosen so that the ordered triple consisting of the tangent vector, the principal normal vector, and the positive $z$-direction form a right hand system. The curvature may be positive or negative. By use of the Frenet formula for plane curves and the fact that the principal normals of the $p_{k}, t_{k}$ congruences lie along $t_{k},-p_{k}$, respectively, we find

$$
\begin{aligned}
p^{i} \partial_{i} p_{k} & =w_{k}=k^{\prime} t_{k}, \\
t^{i} \partial_{j} t_{k} & =m_{k}=-k p_{k},
\end{aligned}
$$

where $k^{\prime}$ and $k$ are the curvatures of the curves, $\beta=$ variable and $\alpha=$ variable, respectively. From the equations (5.2), (5.5), it follows that

$$
k^{\prime}=\frac{t^{i}}{B} \frac{\partial p_{i}}{\partial \beta}, \quad-k=\frac{p^{i}}{A} \frac{\partial t_{i}}{\partial \alpha} .
$$

Substituting (5.3) into (5.6), we find that

$$
k^{\prime}=\frac{1}{B} \frac{\partial \theta}{\partial \beta}, \quad k=\frac{1}{A} \frac{\partial \theta}{\partial \alpha} .
$$

Again, we note that the integrability conditions of (5.4) are

$$
\frac{\partial \theta}{\partial \alpha}=\frac{1}{B} \frac{\partial A}{\partial \beta}, \quad \frac{\partial \theta}{\partial \beta}=-\frac{1}{A} \frac{\partial B}{\partial \alpha} .
$$

The relations (5.5), (5.7), lead to the useful results 


$$
\begin{aligned}
& w_{k} t^{k}=k^{\prime}=-\frac{1}{A B} \frac{\partial B}{\partial \alpha}, \\
& m_{k} p^{k}=-k=-\frac{1}{A B} \frac{\partial A}{\partial \beta}
\end{aligned}
$$

By use of (5.4), we see that the matrix of the transformation (5.1) is

$$
\left[\begin{array}{lll}
\frac{\partial x}{\partial \alpha} & \frac{\partial y}{\partial \alpha} & \frac{\partial z}{\partial \alpha} \\
\frac{\partial x}{\partial \beta} & \frac{\partial y}{\partial \beta} & \frac{\partial z}{\partial \beta} \\
\frac{\partial x}{\partial \delta} & \frac{\partial y}{\partial \delta} & \frac{\partial z}{\partial \delta}
\end{array}\right]=\left[\begin{array}{ccc}
A \cos \theta & A \sin \theta & 0 \\
B \sin \theta & -B \cos \theta & 0 \\
\frac{\partial x}{\partial \delta} & \frac{\partial y}{\partial \delta} & 1
\end{array}\right]
$$

Since the matrix of $\partial \alpha / \partial x$ etc. consists of the reduced cofactors of the determinant of the matrix (5.9), we find

$$
\left[\begin{array}{lll}
\frac{\partial \alpha}{\partial x} & \frac{\partial \beta}{\partial x} & \frac{\partial \delta}{\partial x} \\
\frac{\partial \alpha}{\partial y} & \frac{\partial \beta}{\partial y} & \frac{\partial \delta}{\partial y} \\
\frac{\partial \alpha}{\partial z} & \frac{\partial \beta}{\partial z} & \frac{\partial \delta}{\partial z}
\end{array}\right]=\left[\begin{array}{ccc}
\frac{\cos \theta}{A} & \frac{\sin \theta}{B} & 0 \\
\frac{\sin \theta}{A} & -\frac{\cos \theta}{B} & 0 \\
-a_{13} & -a_{23} & 1
\end{array}\right]
$$

where

$$
\begin{aligned}
& a_{13}=\frac{\sin \theta}{A} \frac{\partial y}{\partial \delta}+\frac{\cos \theta}{A} \frac{\partial x}{\partial \delta} \\
& a_{23}=-\frac{\cos \theta}{B} \frac{\partial y}{\partial \delta}+\frac{\sin \theta}{B} \frac{\partial x}{\partial \delta} .
\end{aligned}
$$

Thus, we find by use of the chain rule and (5.10)

$$
\left.\frac{\partial}{\partial z}\right)_{z, y}=-a_{18} \frac{\partial}{\partial \alpha}-a_{23} \frac{\partial}{\partial \beta}+\frac{\partial}{\partial \delta} .
$$

The significance of the coefficients $a_{13}, a_{23}$ can be seen from the following arguments. By use of (5.9), (5.11), we find that

$$
\begin{aligned}
& a_{13}=\frac{1}{A^{2}}\left(\frac{\partial x}{\partial \alpha} \frac{\partial x}{\partial \delta}+\frac{\partial y}{\partial \alpha} \frac{\partial y}{\partial \delta}+(0)(1)\right) \\
& a_{23}=\frac{1}{B^{2}}\left(\frac{\partial x}{\partial \beta} \frac{\partial x}{\partial \delta}+\frac{\partial y}{\partial \beta} \frac{\partial y}{\partial \delta}+(0)(1)\right)
\end{aligned}
$$

If $\cos (\alpha, \delta)$ denotes the cosine of the angle between the curves, $\beta=$ constant, $\delta=$ constant, and $\alpha=$ constant, $\beta=$ constant and $\cos (\beta, \delta)$ denotes the cosine of the angle between the curves, $\alpha=$ constant, $\delta=$ constant and $\alpha=$ constant, $\beta=$ variable then

$$
A a_{13}=\cos (\alpha, \delta), \quad B a_{23}=\cos (\beta, \delta) \text {. }
$$


Again, we note that if we solve (5.11) for $\partial y / \partial \delta, \partial x / \partial \delta$, we obtain

$$
\begin{aligned}
& \frac{\partial x}{\partial \delta}=B a_{23} \sin \theta+A a_{13} \cos \theta, \\
& \frac{\partial y}{\partial \delta}=A a_{13} \sin \theta-B a_{23} \cos \theta .
\end{aligned}
$$

From (5.4), (5.15), we obtain a set of integrability conditions which must be added to the equations (5.7). These are

$$
\begin{aligned}
& \frac{\partial}{\partial \alpha}\left(B a_{23}\right)-A a_{13} \frac{\partial \theta}{\partial \alpha}=-A \frac{\partial \theta}{\partial \delta}, \\
& \frac{\partial}{\partial \alpha}\left(A a_{13}\right)+B a_{23} \frac{\partial \theta}{\partial \alpha}=\frac{\partial A}{\partial \delta}, \\
& \frac{\partial}{\partial \beta}\left(B a_{23}\right)-A a_{13} \frac{\partial \theta}{\partial \beta}=\frac{\partial B}{\partial \delta}, \\
& \frac{\partial}{\partial \beta}\left(A a_{13}\right)+B a_{23} \frac{\partial \theta}{\partial \beta}=B \frac{\partial \theta}{\partial \delta} .
\end{aligned}
$$

From (5.14), we see that if the angles between the coordinate lines, $\alpha=$ variable and $\delta=$ variable, $\beta=$ variable and $\delta=$ variable, are $\pi / 2$, then $A a_{13}=B a_{23}=0$. The above relations show that in this case, $\theta, A, B$ are all independent of $\delta$ (as is to be expected). The relations (5.7), (5.16) through (5.19) imply that the Riemann tensor vanishes in the $\alpha, \beta, \delta$ system.

To determine formulas (4.1), (4.5), (4.6), (3.6) in terms of the congruences $t^{i}, p^{i}, n^{j}$, we must evaluate the curvature terms. Since, the $n^{i}$ congruence consists of straight lines parallel to the z-axis, the curvature vector of these curves, $u_{k}$, vanishes. Further, the second fundamental tensor of the planes $\left(z=\right.$ constant) is $s_{i k}=0$; also, the mean curvature, $M^{*}$ of these planes vanishes. Again, we consider the curvature, $K$, of (3.10)

$$
K=n^{i} p^{k}\left(\partial_{i} t_{k}-\partial_{k} t_{j}\right) .
$$

Since the vector $t_{i}$ lies in the plane, $z=$ constant

$$
n^{i} p^{k} \partial_{k} t_{j}=0 .
$$

Further, $n^{i} \partial_{j} t_{k}$ represents the directional derivative of $t_{k}$ in the $z$-direction. From geometric considerations, or use of (5.3), it follows that

$$
\left.n^{j} \partial_{i} t_{k}=-\frac{\partial \theta}{\partial z}\right)_{x, y} p_{k} .
$$

By use of the above results and (5.12), the formula for $K$ becomes

$$
K=\left(a_{13} \frac{\partial \theta}{\partial \alpha}+a_{23} \frac{\partial \theta}{\partial \beta}-\frac{\partial \theta}{\partial \delta}\right) .
$$

Finally, the directional derivatives $\partial / \partial t, \partial / \partial n, \partial / \partial p$ become 


$$
\begin{aligned}
& \frac{\partial}{\partial t}=A^{-1} \frac{\partial}{\partial \alpha}, \quad \frac{\partial}{\partial p}=B^{-1} \frac{\partial}{\partial \beta} \\
& \left.\frac{\partial}{\partial n}=\frac{\partial \mathrm{l}}{\partial z}\right)_{x, y}=-\left(a_{13} \frac{\partial}{\partial \alpha}+a_{23} \frac{\partial}{\partial \beta}-\frac{\partial}{\partial \delta}\right) .
\end{aligned}
$$

Now, we express the system (4.1), (4.5), (4.6), (3.6) in terms of the metric quantities $A, B, a_{12}, a_{13}, \theta$, and the partial derivatives $\partial / \partial \alpha, \partial / \partial \beta, \partial / \partial \delta$. By use of (5.8), (5.21), we find that (4.1) becomes

$$
b \frac{\partial}{\partial \alpha} \frac{q^{2}}{b}=c^{2} \frac{\partial}{\partial \alpha} \ln B+\frac{\partial h_{0}}{\partial \alpha}-T \frac{\partial S}{\partial \alpha} .
$$

Further, by use of (5.21), (5.8), relation (4.5) reduces to

$$
\begin{aligned}
-b\left[(\gamma-3) q^{2}+4 c^{2}\right]\left[a_{13} \frac{\partial b}{\partial \alpha}+a_{23} \frac{\partial b}{\partial \beta}-\frac{\partial b}{\partial \delta}\right] \\
=(\gamma-1) b^{3} c A^{-1} \frac{\partial}{\partial \alpha} \ln B-\left[(\gamma-3) q^{2}-(\gamma-5) c^{2}\right] \\
\cdot\left[a_{13} \frac{\partial h_{0}}{\partial \alpha}+a_{23} \frac{\partial h_{0}}{\partial \beta}-\frac{\partial h_{0}}{\partial \delta}\right]-T\left[2 q^{2}-(\gamma+3) c^{2}\right] \\
\cdot\left[a_{13} \frac{\partial S}{\partial \alpha}+a_{23} \frac{\partial S}{\partial \beta}-\frac{\partial S}{\partial \delta}\right] .
\end{aligned}
$$

By use of (5.20), (5.21), (5.8), we find that (4.6) becomes

$$
q \frac{\partial q}{\partial \beta}=\frac{\partial h_{0}}{\partial \beta}-T \frac{\partial S}{\partial \beta}-b^{2} \frac{\partial}{\partial \beta} \ln A+c b B\left[a_{13} \frac{\partial \theta}{\partial \alpha}+a_{23} \frac{\partial \theta}{\partial \beta}-\frac{\partial \theta}{\partial \delta}\right]
$$

Finally, (3.6) reduces to

$$
\left(-c a_{13}+b A^{-1}\right) \frac{\partial h_{0}}{\partial \alpha}-c a_{23} \frac{\partial h_{0}}{\partial \beta}+c \frac{\partial h_{0}}{\partial \delta}=0 .
$$

From (5.25), it follows that the differential equations of the stream lines are

$$
\frac{A d \alpha}{-A a_{13}+\left(M^{2}-1\right)^{1 / 2}}=\frac{d \beta}{-a_{23}}=d \delta
$$

where $M$ is the Mach number, $q / c$. Since $A d \alpha, B d \beta, d \delta$ are the arc length elements along the coordinate lines and $A a_{13}, B a_{23}$ are $\cos (\alpha, \delta), \cos (\beta, \delta)$, it follows from (5.26) that in general the stream lines are space curves.

6. A family of isentropic Beltrami flows with plane characteristic surfaces (helical stream lines, concentric circular bicharacteristics). We shall consider those flows of Sec. 5 for which

$$
h_{0}=\text { constant, } S=\text { constant, } a_{13}=a_{23}=0 \text {. }
$$

From (3.4), it follows that in any isentropic flow for which $h_{0}$ is constant, the vorticity vector is parallel to the veloctiy vector. Such flows are called Beltrami flows and are more general than irrotational flows. As noted earlier, (5.16) through (5.19) imply that 


$$
\frac{\partial A}{\partial \delta}=\frac{\partial B}{\partial \delta}=\frac{\partial \theta}{\partial \delta}=0
$$

that is, the curves, $\delta=$ variable, are orthogonal to the planes, $z=$ constant. Stated in other words, every family of $\infty^{1}$ bicharacteristics $(\delta=$ variable, $\alpha=$ variable) forms surfaces with straight line generators parallel to the z-axis; also, every family of $\infty^{1}$ orthogonal trajectories of the bicharacteristics ( $\delta=$ variable, $\beta=$ variable) forms similar surfaces. From (5.23), (6.1), (6.2), we see by use of a simple argument that

$$
\frac{\partial q}{\partial \delta}=\frac{\partial B}{\partial \alpha}=0
$$

From (6.2), (6.3), we see that, $B=1$, by proper choice of scale factor along the $\beta=$ variable curves. Further, (5.7) implies that the orthogonal trajectories of the bicharacteristics are straight lines or $k^{\prime}=0, \theta=\theta(\alpha)$ and

$$
A=\beta \theta^{\prime}+f(\alpha) \text {, }
$$

where $\theta^{\prime}=d \theta / d \alpha$ and $f(\alpha)$ is an arbitrary function of $\alpha$. Returning to (5.22), (5.23), (5.24), we see that (6.1), (6.2) lead to

$$
\begin{gathered}
q=q(\beta), \\
\frac{M^{2}}{2\left(M^{2}-1\right)} \frac{d}{d \beta} \ln \frac{M^{2}}{2+(\gamma-1) M^{2}}=-\frac{\partial}{\partial \beta} \ln A,
\end{gathered}
$$

where $M=q / c$ is the Mach number. Since $M$ is a function of only $\beta$, we see that in (6.4)

$$
\theta(\alpha)=c_{1} \alpha, \quad f(\alpha)=c_{2} c_{1},
$$

where $c_{1}, c_{2}$ are arbitrary constants. Integrating (6.6) and choosing a proper scale factor along the $\alpha=$ variable curves, we obtain

$$
\frac{2+(\gamma-1) M^{2}}{M^{2}-1}=A^{(\gamma+1)}
$$

To interpret (6.8), we must determine the significance of $A$. From (6.7), (6.4) we find

$$
A=c_{1}\left(\beta+c_{2}\right) \text {. }
$$

Since, $B=1$, we obtain from (5.4)

$$
x=\left(\beta+c_{2}\right) \sin \theta, \quad y=-\left(\beta+c_{2}\right) \cos \theta .
$$

From (6.9), we find that, $c_{2}+\beta=r$, where $r$ is the radial distance variable in polar coordinates. If we introduce the polar angle $\phi=\theta-\pi / 2$ into (6.9) then

$$
x=\mathrm{r} \cos \phi, \quad y=r \sin \phi .
$$

Thus, (6.8), may be written as

$$
\frac{2+(\gamma-1) M^{2}}{M^{2}-1}=C r^{(\gamma+1)}
$$

where $C$. is a constant. It is easily shown from (6.11) that for $\gamma>-1, M$ decreases as $r$ increases, $M=1$ as $r$ approaches infinity, $M$ approaches infinity when $r=[(\gamma-1) / C]^{1 /(\gamma+1)}$. 
From (6.9), we see that the bicharacteristics, $r=c_{2}+\beta=$ constant, are concentric circles. By integrating (5.26), we obtain

$$
\phi=\frac{\left(M^{2}-1\right)^{1 / 2}}{r} z+c_{8}, \quad r=\text { constant }
$$

where $c_{3}$ is an arbitrary constant. Thus, the stream lines are helices and cut the generators of the cylinder, $r=$ constant, in the constant Mach angle $\psi$, where

$$
\tan \psi=\left(M^{2}-1\right)^{1 / 2} \text {. }
$$

By use of (3.3), and the relations (see 5.5)

$$
K=u_{k}=s_{i k}=\frac{\partial c}{\partial t}=\frac{\partial b}{\partial n}=0, \quad p^{k} m_{k}=-\frac{1}{r}
$$

we find that

$$
\omega^{i}=-\frac{d c}{d r} t^{i}+\left(\frac{d b}{d r}+\frac{b}{r}\right) n^{i}
$$

Thus, the present class of flows are rotational flows. Evidently $q$ is constant along each stream line [8].

By assigning various appropriate values to $a_{13}, a_{23}$, other flows may be obtained.

\section{BibLIOGRAPHY}

1. N. Coburn, Intrinsic relations satisfied by the velocity and vorticity vectors, Michigan Math. J. (2) 1, 113-130 (1952)

2. N. Coburn, Discontinuities in compressible fluid flows, Math. Mag. (5) 27, 245-264 (1954). The extensions to the non-isentropic flow of a polytropic gas and a general fluid were obtained by Mr. J. McCully and Mr. L. E. Miller, respectively (not published as yet)

3. R. Courant and K. O. Friedrichs, Supersonic flow and shock waves, Interscience Publishers, N.Y., 1948, p. 247 for the isentropic two-dimensional case

4. C. L. Dolph and N. Coburn, The method of characteristics in the three-dimensional stationary supersonic flow of a compressible gas, Proc. of the First Symposium of Appl. Math., 1947, Am. Math. Soc., 1949, 55-66

5. N. Coburn, Vector and tensor analysis, The Macmillan Co., N.Y., 1955, p. 227

6. Reference 3, p. 22

7. Reference 5, p. 294

8. R. H. Wasserman in some recent work on his doctorate thesis has classified all flows with helical stream lines and has verified the existence of the flow of Sec. 6 